\title{
Shensong Yangxin capsules prevent ischemic arrhythmias by prolonging action potentials and alleviating $\mathrm{Ca}^{2+}$ overload
}

\author{
YIXIU ZHAO ${ }^{1,2}$, FENG GAO $^{1}$, YONG ZHANG ${ }^{1,2}$, HONGTAO WANG $^{3}$, \\ JIUXIN ZHU ${ }^{1,2}$, LIPING CHANG ${ }^{4}$, ZHIMIN DU ${ }^{5}$ and YAN ZHANG ${ }^{1,2}$ \\ ${ }^{1}$ Department of Pharmacology, ${ }^{2}$ Key Laboratory of Cardiovascular Medicine Research, Harbin Medical University, \\ Harbin, Heilongjiang 150081; ${ }^{3}$ State Key Laboratory of Collateral Disease Research and Innovation Medicine, \\ Hebei Yiling Pharmaceutical Research Institute; ${ }^{4}$ Graduate School, Hebei Medical University, Shijiazhuang, Hebei 050000; \\ ${ }^{5}$ Department of Clinical Pharmacy, The Second Affiliated Hospital of Harbin Medical University, \\ Harbin, Heilongjiang 150086, P.R. China
}

Received May 28,2015; Accepted April 7, 2016

DOI: $10.3892 / \mathrm{mmr} .2016 .5203$

\begin{abstract}
Shensong Yangxin capsules (SSYX) are an effective traditional Chinese medicine that has been used to treat coronary heart disease clinically. The present study aimed to establish whether SSYX prevent ischemic arrhythmias in rats, and to explore the underlying mechanisms. Male rats were pretreated with distilled water, SSYX and amiodarone for one week. Acute myocardial ischemia (AMI) was performed to induce ischemic arrhythmias. The incidence and severity of ischemic arrhythmias were evaluated. The action potential, transient outward $\mathrm{K}^{+}$current $\left(\mathrm{I}_{\mathrm{to}}\right)$ and inward rectifier $\mathrm{K}^{+}$ current $\left(\mathrm{I}_{\mathrm{K} 1}\right)$ of rat cardiomyocytes were measured using the patch-clamp technique. The intracellular $\mathrm{Ca}^{2+}$ concentration of the cardiomyocytes was measured using a laser scanning confocal microscope. The results revealed that SSYX lowered the incidence of arrhythmia markedly during AMI. Furthermore, SSYX delayed the appearance, and reduced the severity, of ischemic arrhythmias compared with the control. In addition, SSYX markedly decreased the ratio of the myocardial infarction region to the whole heart. In an in vitro study, SSYX prolonged the action potential duration of rat cardiomyocytes, and inhibited $I_{t o}$ and $I_{K 1}$ markedly. Additionally, SSYX inhibited $\mathrm{Ca}^{2+}$ elevation induced by $\mathrm{KCl}$ in cardiomyocytes. These results suggested that SSYX prevents ischemic arrhythmia, and the underlying mechanism responsible for this process may include prolonging the action potential and alleviating $\mathrm{Ca}^{2+}$ overload.
\end{abstract}

Correspondence to: Professor Yan Zhang, Department of Pharmacology, Harbin Medical University, 157 Baojian Road, Nangang, Harbin, Heilongjiang 150081, P.R. China

E-mail: zhangyan@ems.hrbmu.edu.cn

Key words: ventricular arrhythmias, transient outward $\mathrm{K}^{+}$current, inward rectifier $\mathrm{K}^{+}$current, action potential duration, $\mathrm{Ca}^{2+}$ overload, serum pharmacology

\section{Introduction}

Despite numerous previous advances in the treatment of cardiovascular disease, sudden cardiac death caused by ischemic arrhythmias remains a major reason of mortality worldwide (1). Numerous intensive studies have been conducted on researching and developing drugs to treat arrhythmias over the course of the last few past decades, and only a few of them have assisted in enabling patients to live longer whereas, in effect, the majority of them may have led to mortality due to their proarrhythmic potentials (2). The underlying disease causing ischemic arrhythmias is usually atherosclerotic coronary artery disease, which narrows or occludes the coronary artery and induces myocardial ischemia and infarction (3). A previous study has shown that various mechanisms trigger ischemic arrhythmias during myocardial ischemia, including reconstruction of the cardiac sympathetic nerve, alterations of the transmembrane ion current and intracellular $\mathrm{Ca}^{2+}$ $\left(\left[\mathrm{Ca}^{2+}\right]_{\mathrm{i}}\right)$ dysregulation (4). Myocardial ischemia affects the electrophysiological properties of the cardiac cells, inducing the generation and conduction of abnormal impulses, reducing the fibrillation threshold and initiating ectopic impulses. These ectopic beats cause abnormal current flow across the boundary between ischemic and non-ischemic myocardium (5). In addition, $\mathrm{Ca}^{2+}$ overload during the myocardial ischemia diastolic period causes afterdepolarizations and aftercontractions, which are the primary causes of electrical instability (6). Changes in the resting potential and the inward and outward currents of the action potentials lead to alterations in conduction, refractoriness and automaticity, all of which contribute to the occurrence of ischemic arrhythmias.

Traditional Chinese medicines (TCMs) have been extensively used to treat arrhythmias due to their stable curative effects and low toxicity. In addition, TCMs mediate their effects via different pathways and multiple targets simultaneously, due to their various components. Shensong Yangxin capsules (SSYX) are clinically used to treat coronary heart disease (7). The bioactive components of SSYX include sodium danshensu, chlorogenic acid, paeoniflorin, spinosin, salvianolic acid $B$, berberine hydrochloride, gensenoside $R b_{1}$ 
and schisantherin A $(8,9)$. Several studies demonstrated that SSYX inhibited multiple ion channels in cardiac cells, including the potassium/sodium hyperpolarization-activated cyclic nucleotide-gated channel 4 (HCN4) and L-type calcium channel, which may affect the electrical activity of myocardial cells and contribute to their antiarrhythmic effects (10-12). The present study aimed to establish whether SSYX prevents ischemic arrhythmias, and to explore the underlying mechanisms.

\section{Materials and methods}

Establishment of ischemic arrhythmias. SSYX (cat. no. Z20030058; Yiling Phamaceutical Co., Ltd., Shijiazhuang, China) and amiodarone (cat. no. P20080122150958640; Harvest Pharmaceutical Co., Ltd., Shanghai, China) were dissolved in distilled water and administered to rats (13). Amiodarone was used for the purposes of comparison. All experiments were approved by the ethics committee of Harbin Medical University, and the health guide for the use of experimental animals of the National Institutes was observed. Sprague-Dawley rats (male; $180 \pm 20$ g; 6-8 weeks) were purchased from the Animal Center of the Second Affiliated Hospital of Harbin Medical University (certificate no. SCXK (Ha) 2012-010). The animals were housed under standard laboratory conditions (temperature, $20-22^{\circ} \mathrm{C}$; humidity, $40-70 \% ; 12 \mathrm{~h} / 12 \mathrm{~h}$ day-night cycle) and divided into four groups at random: Sham $(n=8)$ rats had the chest opened without ligating the coronary artery; for the control $(n=8)$ rats, distilled water was administered intragastrically once a day for 7 days prior to ligation of the coronary artery; for the SSYX $(\mathrm{n}=8)$ rats, a suspension of SSYX $(1.8 \mathrm{~g} / \mathrm{kg})$ was administered to rats once a day for 7 days intragastrically prior to ligation of the coronary artery; and for the amiodarone $(n=8)$ rats, a suspension of amiodarone $(0.12 \mathrm{~g} / \mathrm{kg})$ was administered intragastrically once a day for 7 days prior to ligation of the coronary artery.

Ischemic arrhythmias induced by acute myocardial ischemia (AMI) were performed as previously described $(14,15)$. At $1 \mathrm{~h}$ following the final administration of the respective drugs, rats were anesthetized with $3 \%$ sodium pentobarbital $(0.12 \mathrm{ml} / 100 \mathrm{~g})$, and the left anterior descending branch of the coronary artery was subsequently ligated. Electrocardiograms and the heart rate were monitored using the BL-420F Data Acquisition and Analysis system (TME Technology Co., Ltd, Chengdu, China). Evident S-T elevation, as shown in the electrocardiogram, and a darkened myocardium under the ligature indicated that the AMI model had been successfully constructed. The incidence and onset of arrhythmias were monitored on the electrocardiogram. The arrhythmia score was calculated to assess the severity of arrhythmias, according to Lambeth Conventions (16). The infarct region was measured by Evans Blue (Sigma-Aldrich, St Louis, USA) staining and 2,3,5-triphenyltetrazolium chloride (Sigma, St Louis, USA) staining after ligation for $6 \mathrm{~h}$. The area dyed grey was defined as the infarction area, whereas the red coloration indicated ischemic tissue, and blue indicated the presence of normal myocardium (17). The infarction area of each heart was cut and weighed to calculate the ratio of the myocardial infarction region to the whole heart.
Whole-cell patch-clamp recording

Serum preparation. Serum containing SSYX was prepared according to a previously described method (18). Distilled water and suspensions of SSYX were administered to rats by gavage $(1.8 \mathrm{~g} / \mathrm{kg})$ once a day for 7 days. At $2 \mathrm{~h}$ following the final administration, the blood was collected from the abdominal aorta of rats and maintained for $1 \mathrm{~h}$ at $25^{\circ} \mathrm{C}$. Subsequently, the blood was centrifuged at $3,000 \mathrm{x}$ g for $10 \mathrm{~min}$ to separate the serum. The serum was then centrifuged at 3,000 $\mathrm{x} g$ for $30 \mathrm{~min}$ in a $3 \mathrm{kDa}$ tube (Millipore Corp., Billerica, MA, USA) to remove proteins. The filtrate was dried into serum powder sing a pressure blowing concentrator (TTL-DCI model; Beijing Tongtailian Science and Technology Development Co., Ltd., Beijing, China). Subsequently, serum samples of the control and SSYX rats were subjected to high-pressure liquid chromatography (HPLC) analysis. The analysis was performed with a 5C18-MS-II packed column (model 38020-41; 250x4.6 mm; $5 \mu \mathrm{m}$; Cosmosil, Tokyo, Japan) maintained at $30^{\circ} \mathrm{C}$ under isocratic flow conditions. The mobile phase was $80 \%$ methanol, and the flow rate was $1 \mathrm{ml} / \mathrm{min}$. SSYX serum was monitored at $254 \mathrm{~nm}$ and identified using the LC-20A HPLC system of Shimadzu (Shimadzu, Tokyo, Japan).

Separation and isolation of cardiomyocytes. Cardiomyocytes were isolated from the rat hearts by enzymatic dissociation (19). Hearts were taken out from the chest of the rats following anesthetization with sodium pentobarbital, and reversely perfused through the aorta using a modified Langendorff perfusion system with $\mathrm{Ca}^{2+}(-)$ Tyrode's solution containing (in $\mathrm{mM}$ ): $\mathrm{NaCl}, 126.0 ; \mathrm{KCl}, 5.4 ; \mathrm{MgCl}_{2}, 1$; $\mathrm{NaH}_{2} \mathrm{PO}_{4}, 0.33$; glucose, 10 and Hepes, 10, at pH 7.4 for 5 min. $\mathrm{Ca}^{2+}(-)$ Tyrode's solution was oxygenated and maintained at $37^{\circ} \mathrm{C}$ prior to the experiment. Subsequently, perfusate containing $160 \mathrm{mg} / \mathrm{l}$ collagenase II (Wako, Osaka, Japan) and $160 \mathrm{mg} / \mathrm{l}$ bovine serum albumin (Sigma-Aldrich) was used to digest myocardium continuously for $\sim 20 \mathrm{~min}$. After the heart had become soft and light, the left ventricular tissues were cut up and agitated gently in a test tube. Isolated single cardiomyocytes were stored in preservation solution containing (in $\mathrm{mM}$ ): glutamic acid, 70; taurine, $15 ; \mathrm{KCl}, 30 ; \mathrm{KH}_{2} \mathrm{PO}_{4}, 10$; $\mathrm{MgCl}_{2}, 0.5$; ethylene glycol-O-O'-bis(2-amino-ethyl)-N, $\mathrm{N}^{\prime} \mathrm{N}^{\prime}$, $\mathrm{N}^{\prime}$-tetraacetic acid (EGTA), 0.5; Hepes, 10; and glucose, 10, at $\mathrm{pH} 7.4$ and at room temperature for later use.

Measurement of the action potential, transient outward $K^{+}$current $\left(I_{t o}\right)$ and inward rectifier $K^{+}$current $\left(I_{K I}\right)$. Blank and SSYX sera were dissolved in $20 \% \mathrm{Ca}^{2+}$ Tyrode's solution. The action potential duration (APD), $I_{t o}$ and $I_{K 1}$ in rat cardiomyocytes were recorded using the patch-clamp technique (20-22). Cells were placed in $\mathrm{Ca}^{2+}$ Tyrode's solution in the chamber at room temperature. Borosilicate glass electrodes (Huaxi Medical University Instrument Factory, Chengdu, China) with a tip resistance of $2 \sim 4 \mathrm{M} \Omega$ were filled with the pipette solution containing (in $\mathrm{mM}$ ): $\mathrm{KCl}, 20$; potassium aspartate, 110; $\mathrm{MgCl}_{2}, 1.0$; Hepes, 5; EGTA, 10; and $\mathrm{Na}_{2} \mathrm{ATP}, 5$ at $\mathrm{pH}$ 7.4. Action potentials were elicited using the current-clamp mode. Cardiomyocytes were electrically stimulated by applying an intracellular depolarizing stimulus (-5 ms duration and $900 \mathrm{pA}$ amplitude) via a digital pulse generator (DD1550A; Axon Instruments, Inc., Foster City, 
A

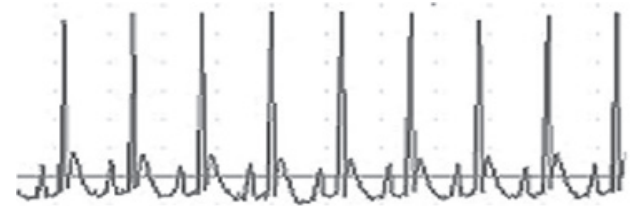

C

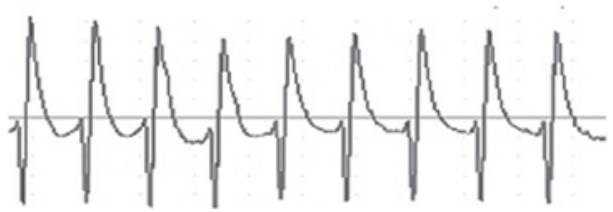

B

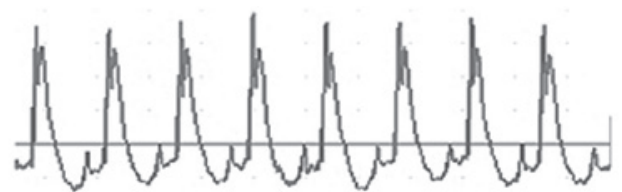

D

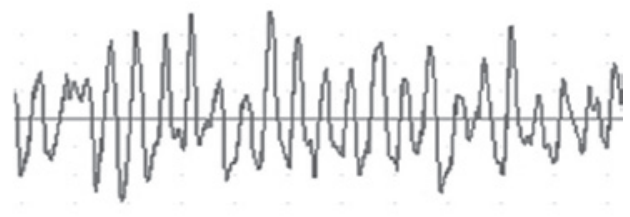

Figure 1. Representative electrocardiograms prior to and following ligation $(\mathrm{n}=8)$. (A) Normal sinus rhythm prior to ligation; (B) S-T elevation during AMI induced by coronary artery occlusion; (C) ventricular tachycardia induced by AMI; (D) ventricular fibrillation induced by AMI. AMI, acute myocardial ischemia.

A

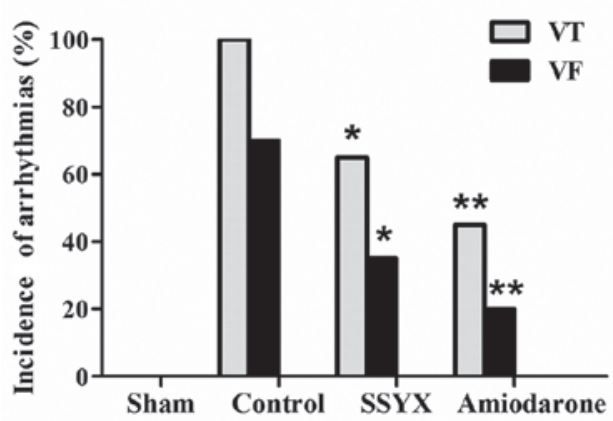

C

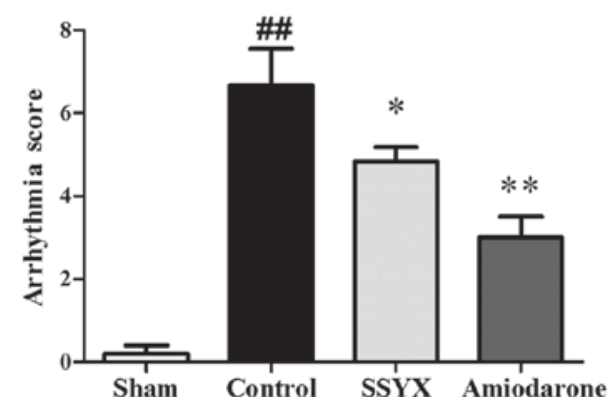

B

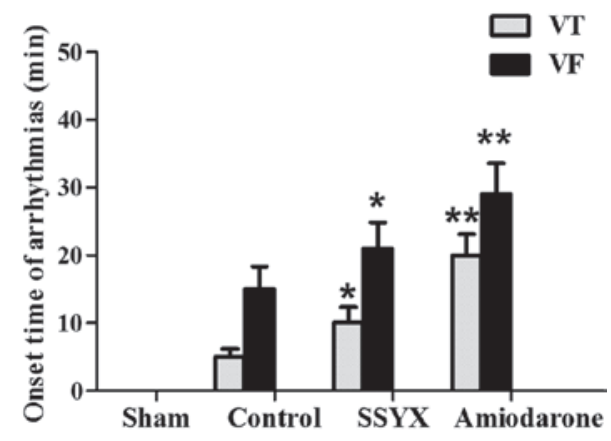

D

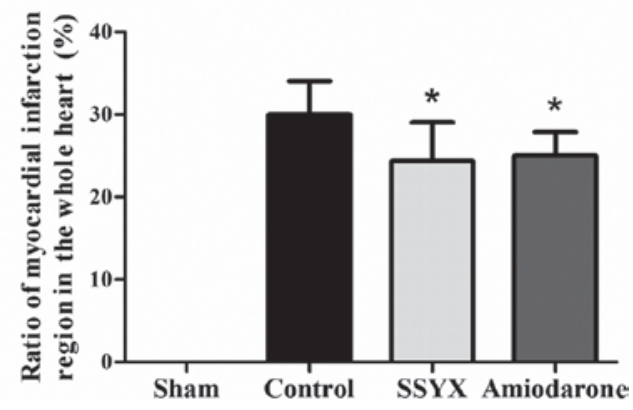

Figure 2. Effects of SSYX on ischemic arrhythmias. (A) SSYX and amiodarone reduced the incidence of VT and VF. (B) SSYX and amiodarone delayed the onset of arrhythmias. (C) SSYX and amiodarone decreased the arrhythmia scores of rats with acute myocardial ischemia. (D) SSYX and amiodarone reduced the ratio of myocardial infarction region to the whole heart. Values are expressed as the mean \pm standard error of the mean. ${ }^{\# \prime} \mathrm{P}<0.01 \mathrm{vs}$. sham, "P<0.05 and ${ }^{* *} \mathrm{P}<0.01$ vs. control. SSYX, Shensong Yangxin capsule; VT, ventricular tachycardia; VF, ventricular fibrillation.

CA, USA). $I_{\text {to }}$ and $I_{K 1}$ were recorded using the voltage-clamp mode. $\mathrm{I}_{\text {to }}$ was evoked by applying $600 \mathrm{~ms}$ pulses with the holding potential of $-40 \mathrm{mV}$, and the test potentials increased from -40 to $+50 \mathrm{mV}$ with a step size of $10 \mathrm{mV}$. $\mathrm{I}_{\mathrm{K} 1}$ was evoked by applying a holding potential of $-40 \mathrm{mV}$ and $500 \mathrm{~ms}$ depolarization. The test potentials increased from -120 to $+50 \mathrm{mV}$ with a step size of $10 \mathrm{mV}$. Transmembrane potentials and ion currents were recorded in the whole-cell recording mode using an Axopatch $^{\mathrm{TM}}$ 200B amplifier (Molecular Devices, LLC, Sunnyvale, CA, USA), and processed using pCLAMP 9.0 software (Axon Instruments, Inc.).

Measurement of $\left[\mathrm{Ca}^{2+}\right]_{i}$. Cardiomyocytes were loaded with $5 \mu \mathrm{M}$ Fluo-3/AM and $10 \mu \mathrm{M}$ Pluronic F-127 (Ambion ${ }^{\circledR}$;
Thermo Fisher Scientific, Waltham, MA, USA). $\left[\mathrm{Ca}^{2+}\right]_{\mathrm{i}}$ was determined according to the changes of fluorescence intensity prior to and following drug administration (23). The effect of SSYX on $\left[\mathrm{Ca}^{2+}\right]_{\mathrm{i}}$ was also studied in cardiomyocytes stimulated with $30 \mathrm{mM} \mathrm{KCl}$. Changes in fluorescence were monitored using a laser scanning confocal microscope (Olympus FV-1000; Olympus Optical Co., Ltd, Tokyo, Japan). The excitation wavelength was set at $488 \mathrm{~nm}$ and the emission wavelength was set at $530 \mathrm{~nm}$. Qualitative changes of $\left[\mathrm{Ca}^{2+}\right]_{\mathrm{i}}$ were indicated as $\mathrm{F}_{\max } / \mathrm{F}_{0}$.

Statistical analysis. Values are expressed as the mean \pm standard error of the mean and analyzed using a two-tailed paired t-test to examine the individual apparent differences. 
$\mathrm{P}<0.05$ was used to indicate a statistically significant difference.

\section{Results}

SSYX prevents ischemic arrhythmias. Electrocardiogram and heart rate were continuously recorded for $30 \mathrm{~min}$ following ligating coronary artery. Electrocardiograms of rats prior to and following ligation are shown in Fig. 1. SSYX and amiodarone significantly reduced the incidence of ventricular tachycardia (VT) and ventricular fibrillation (VF), and delayed the onset of arrhythmias compared with the control (Fig. 2A and B). The severity of ischemic arrhythmias is shown as an arrhythmia score, according to Lambeth Conventions. The arrhythmia score of the control group was significantly higher compared with the sham group $(\mathrm{P}<0.01)$. SSYX and amiodarone reduced the arrhythmia scores of AMI rats compared with the control (Fig. 2C). In addition, SSYX and amiodarone decreased the ratio of the myocardial infarction region to the whole heart (Fig. 2D). All these results indicated that SSYX were able to prevent ischemic arrhythmias.

SSYX prolongs the action potential of rat ventricular myocytes. Myocardial action potentials reflect the process of cardiac depolarization and repolarization. The effect of SSYX on myocardial action potentials was evaluated in rat cardiomyocytes using a serum pharmacological method and the patch-clamp technique. Differences in blank and SSYX serum were then analyzed using HPLC. The results demonstrated that SSYX serum had two significant absorption peaks, which were different from blank serum, suggesting that serum powder containing SSYX had been successfully prepared (Fig. 3). SSYX markedly prolonged the action potential, extending the action potential at $50 \%$ repolarization $\left(\mathrm{APD}_{50} ; 139.29 \pm 45.24 \mathrm{~ms}\right)$ and the action potential at $90 \%$ repolarization $\left(\mathrm{APD}_{90} ; 204.41 \pm 63.66 \mathrm{~ms}\right)$, compared with the control $(84.04 \pm 34.48$ and $150.62 \pm 51.25 \mathrm{~ms}$, respectively). Amiodarone significantly extended $\mathrm{APD}_{50}$ $(181.52 \pm 50.88 \mathrm{~ms})$ and $\mathrm{APD}_{90}(272.14 \pm 73.75 \mathrm{~ms})$ compared with the control (Fig. 4). These results indicated that SSYX was able to extend the action potentials and slow down cardiac repolarization in rapid arrhythmias.

SSYX inhibits $I_{K I}$ and $I_{\text {to }}$ in rat ventricular myocytes. $\mathrm{I}_{\mathrm{to}}$ and $\mathrm{I}_{\mathrm{K} 1}$ are involved in cardiac repolarization, and are associated with cardiac excitability and arrhythmogenesis (24). In this study, the effects of SSYX on $\mathrm{I}_{\mathrm{K} 1}$ and $\mathrm{I}_{\text {to }}$ current were recorded. Fig. 5A and $B$ show the voltage-dependent $I_{K 1}$ current in the presence of blank or SSYX serum (holding potential $-40 \mathrm{mV}$, depolarization pulses $500 \mathrm{~ms}$, test potential increased from -120 to $+50 \mathrm{mV}$, step amplitude $10 \mathrm{mV}$; Fig. 5C). The current-voltage (I-V) curve revealed that SSYX significantly reduced the density of $\mathrm{I}_{\mathrm{K} 1}$. The peak amplitude of $\mathrm{I}_{\mathrm{K} 1}$ was decreased from $-25.04 \pm 2.14$ to $-15.30 \pm 2.05(\mathrm{P}<0.05$; Fig. 5D and E). Fig. 6A and B show the voltage-dependent $\mathrm{I}_{\text {to }}$ current in the presence of blank or SSYX serum (holding potential $-40 \mathrm{mV}, 600 \mathrm{~ms}$ depolarization pulse, test potential increased from -40 to $+50 \mathrm{mV}$, step amplitude $10 \mathrm{mV}$; Fig. 6C) The I-V association for $\mathrm{I}_{\text {to }}$ indicated that SSYX significantly reduced the density of $I_{\text {to }}$ (Fig. 6D). The peak amplitude of
A

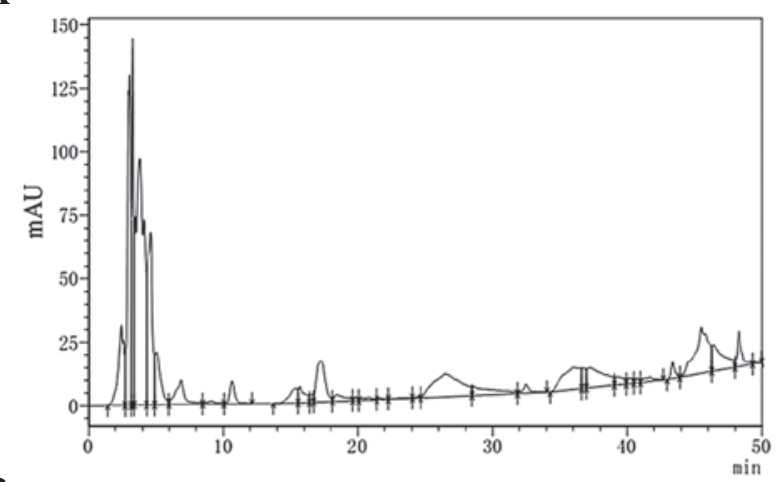

B

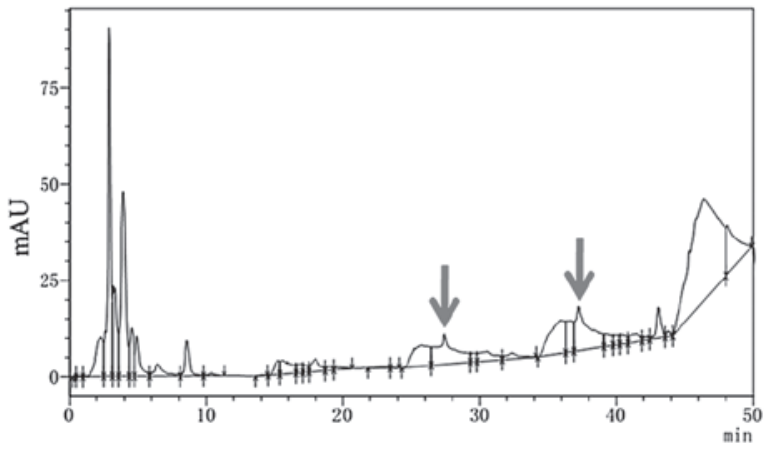

Figure 3. High-performance liquid chromatography analysis of (A) control serum and (B) SSYX-containing serum. The arrows represent the characteristic absorption of SSYX. SSYX, Shensong Yangxin capsule; mAU, milliabsorbance units.

$\mathrm{I}_{\text {to }}$ was decreased from $22.21 \pm 2.04$ to $16.30 \pm 3.00(\mathrm{P}<0.05$; Fig. 6E).

SSYX inhibits $\mathrm{Ca}^{2+}$ overload in rat cardiomyocytes. The effects of SSYX on $\mathrm{Ca}^{2+}$ regulation were further investigated in rat cardiomyocytes loaded with the calcium indicator, Fluo-3/AM. The results demonstrated that the ratio of $\mathrm{F}_{\max } / \mathrm{F}_{0}$ was increased $1.23 \pm 0.19$ times following the addition of SSYX serum and 1.16 \pm 0.16 times after adding blank serum (Fig. 7A). SSYX did not increase the area under the fluorescence intensity curve and the peak fluorescence intensity compared with the control (Fig. 7B and C). Following stimulation with $30 \mathrm{mM}$ $\mathrm{KCl}$, the $\mathrm{F}_{\max } / \mathrm{F}_{0}$ was increased $2.19 \pm 0.37$ times in ventricular myocytes pretreated with SSYX serum, and 3.32 \pm 0.27 times in the control (Fig. 7D). SSYX significantly decreased the area under the fluorescence intensity curve and peak fluorescence intensity compared with the control (Fig. 7E and F). In addition, SSYX decreased the baseline fluorescent intensity compared with the control.

\section{Discussion}

Ventricular arrhythmia remains one of the predominant causes of sudden cardiac death. Prevention and treatment of ventricular arrhythmia are continuing challenges in the medical field. With the increasing incidence of cardiovascular disease, TCMs are becoming more frequently used in China and Western countries due to their preferable efficacies, multiple components and therapeutic targets and safety (25). Clinical studies have shown that SSYX effectively improved 
A

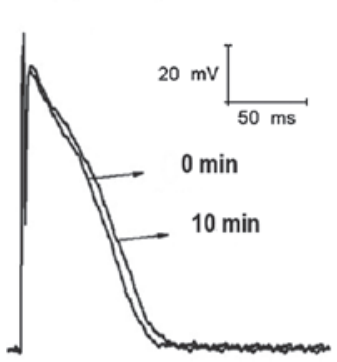

B

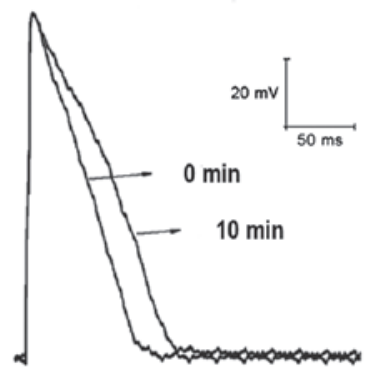

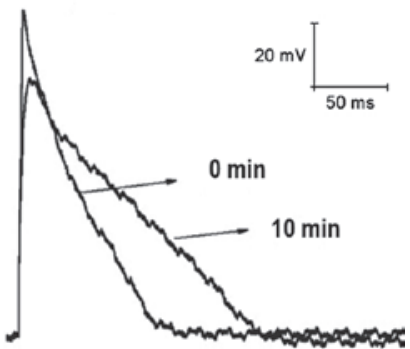

D

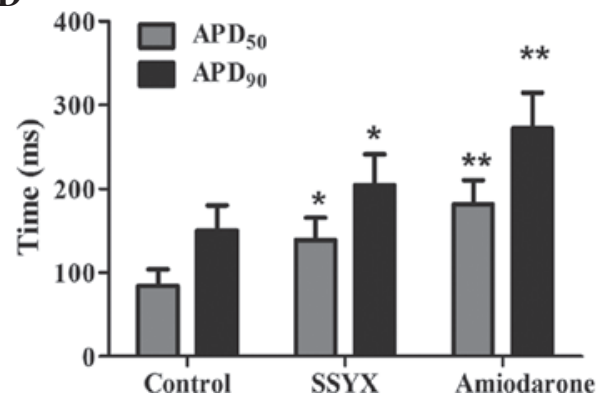

$\mathbf{E}$

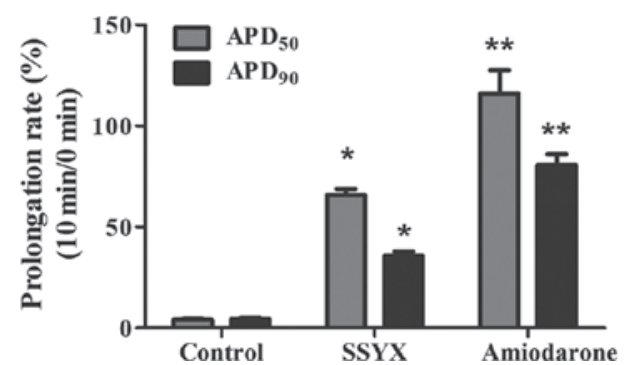

Figure 4. Effects of SSYX on the action potential of cardiomyocytes. (A) Effect of blank serum on the action potential of cardiomyocytes. (B) Effect of SSYX serum on the action potential of cardiomyocytes. (C) Effect of amiodarone on the action potential of cardiomyocytes. (D) APD of cardiomyocytes following treatment with blank serum, SSYX serum or amiodarone. (E) Prolongation of the action potential of cardiomyocytes following treatment with blank serum, SSYX serum or amiodarone. Values are expressed as the mean \pm standard error of the mean. ${ }^{*} \mathrm{P}<0.05$ and ${ }^{* *} \mathrm{P}<0.01$ vs. control. APD, action potential duration; SSYX, Shensong Yangxin capsule.

A

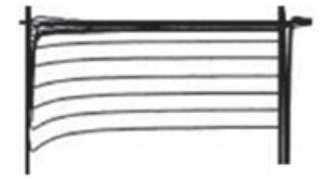

$2000 \mathrm{pA}$
B

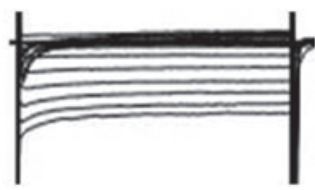

C

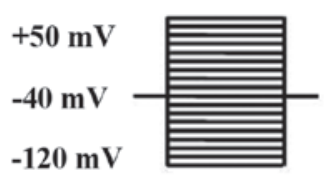

$2000 \mathrm{pA}$

$100 \mathrm{~ms}$

D

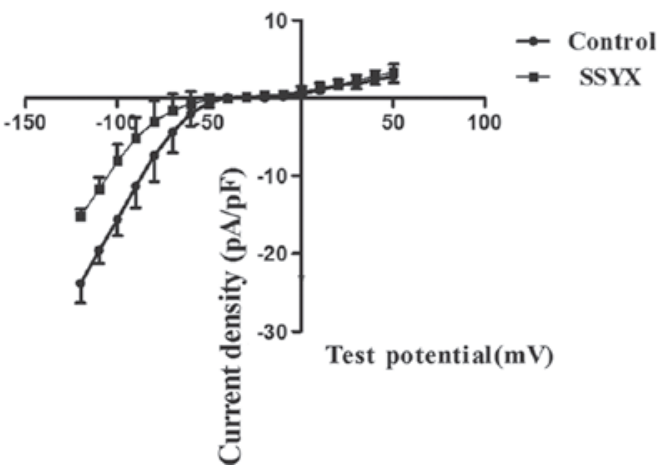

$\mathbf{E}$

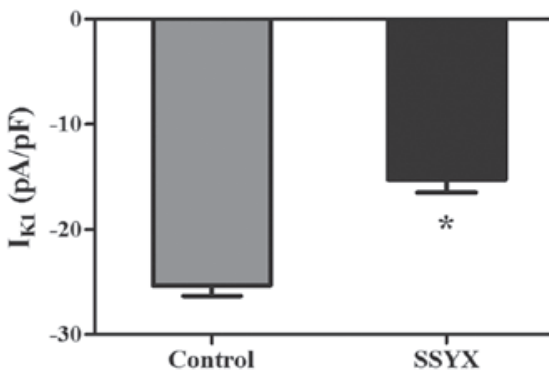

Figure 5. Effects of SSYX on $\mathrm{I}_{\mathrm{K} 1}$. (A) Original traces of $\mathrm{I}_{\mathrm{K} 1}$ in blank serum-treated cells. (B) Original traces of $\mathrm{I}_{\mathrm{K} 1}$ in $\mathrm{SSYX}$ serum-treated cells. (C) Stimulus protocol of $\mathrm{I}_{\mathrm{K} 1}$. (D) Current - voltage curve of $\mathrm{I}_{\mathrm{K} 1}$ in rat cardiomyocytes. (E) $\mathrm{I}_{\mathrm{K} 1}$ densities of blank and SSYX-treated cells at a stimulus voltage of -120 mV. Values are expressed as the mean \pm standard error of the mean. ${ }^{*} \mathrm{P}<0.05$ vs. control. $\mathrm{I}_{\mathrm{K} 1}$, inward rectifier $\mathrm{K}^{+}$current; $\mathrm{SSYX}$, Shensong Yangxin capsule.

heart palpitations, chest tightness, shortness of breath, insomnia and fatigue; these studies also indicated that SSYX effectively suppressed paroxysmal atrial fibrillation and frequent premature ventricular contractions (26-28). The findings of the present study have corroborated that SSYX prevents ischemic arrhythmias, and that this effect was associated with prolonging cardiac action potentials and reducing the $\mathrm{Ca}^{2+}$ overload.
Myocardial ischemia and anoxia disturb cardiac conduction, accelerate cardiac repolarization and cause early afterdepolarization (EAD), which are the primary causes of ischemic arrhythmias (3). Factors, including the heart rate, heart size, size of ischemic area and arrhythmia score, are able to be used to determine the incidence and severity of ischemic arrhythmias. The results in the present study demonstrated that SSYX reduced the incidence of VT and VF, 
A

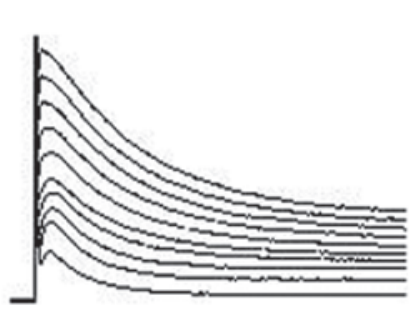

D

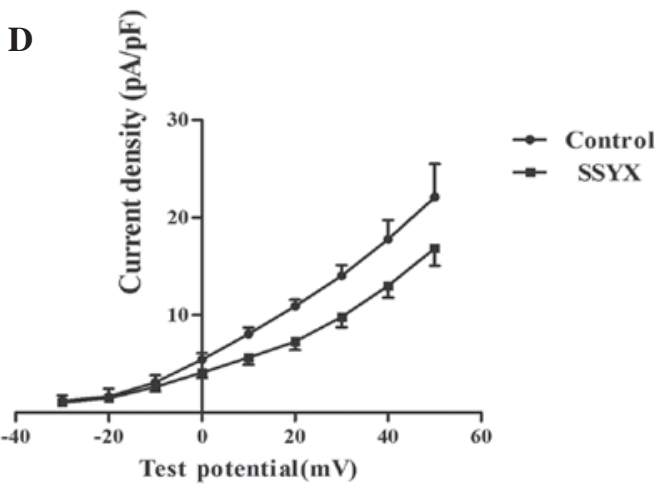

B

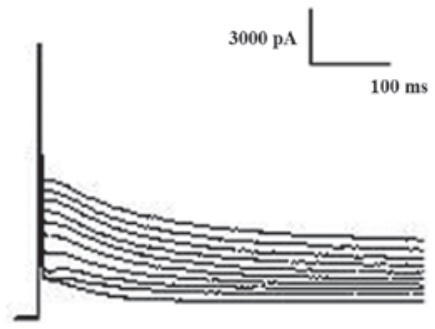

C

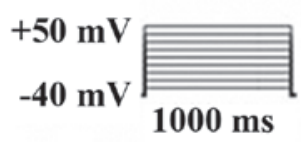

$\mathbf{E}$

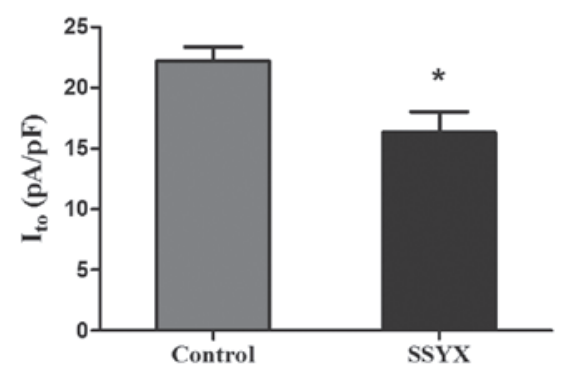

Figure 6. Effects of SSYX on $\mathrm{I}_{\mathrm{to}}$. (A) Original traces of $\mathrm{I}_{\mathrm{to}}$ in blank serum-treated cells. (B) Original traces of $\mathrm{I}_{\mathrm{to}}$ in SSYX serum-treated cells. (C) Stimulus protocol of $\mathrm{I}_{\mathrm{to}}$. (D) Current - voltage curve of $\mathrm{I}_{\mathrm{to}}$ in blank and SSYX-treated rat cardiomyocytes. (E) $\mathrm{I}_{\mathrm{to}}$ densities of blank and SSYX-treated cells at a stimulus voltage of $50 \mathrm{mV}$. "P $<0.05$ vs. control. $\mathrm{I}_{\mathrm{t}}$, transient outward $\mathrm{K}^{+}$current; SSYX, Shensong Yangxin capsule.

A
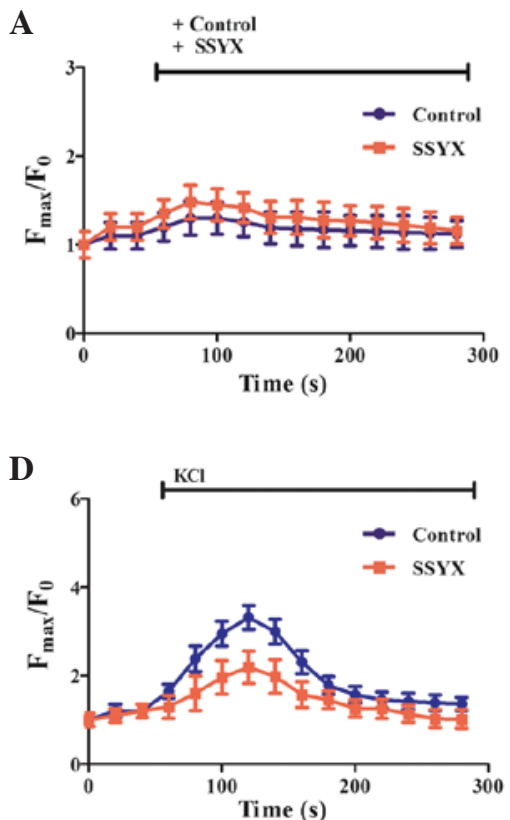

B

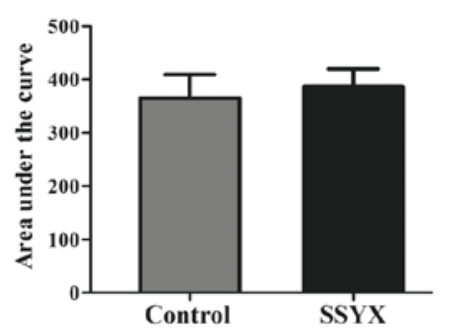

$\mathbf{E}$

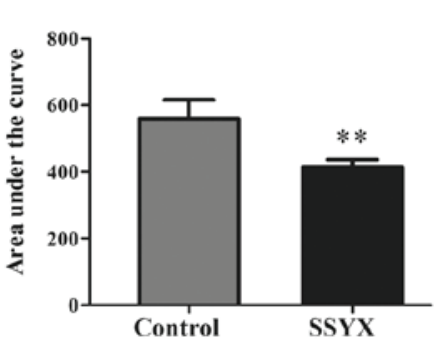

C

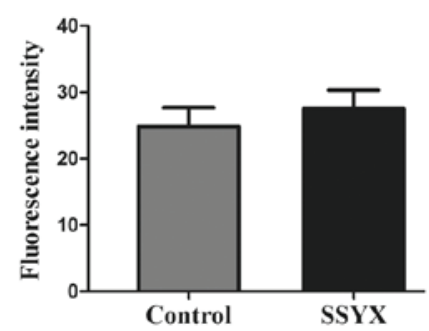

$\mathbf{F}$

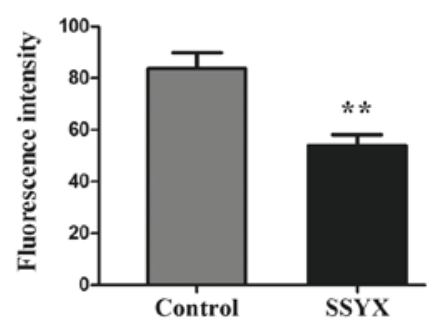

Figure 7. Effect of SSYX on $\mathrm{Ca}^{2+}$ regulation of rat cardiomyocytes. (A) $\mathrm{F}_{\max } / \mathrm{F}_{0}$ of ventricular myocytes following the addition of blank or SSYX serum. (B) SSYX did not increase the area under the fluorescence intensity curve compared with the control. (C) SSYX did not increase the peak fluorescence intensity compared with the control. (D) $\mathrm{F}_{\max } / \mathrm{F}_{0}$ of blank or SSYX serum-treated ventricular myocytes following stimulation with $30 \mathrm{mM} \mathrm{KCl}$. (E) On stimulation with $\mathrm{KCl}$, SSYX significantly decreased the area under the fluorescence intensity curve ( ${ }^{* * *} \mathrm{P}<0.01$ vs. control). (F) On stimulation with $\mathrm{KCl}$, SSYX significantly decreased peak fluorescence intensity ("** $\mathrm{P}<0.01$ vs. control).

and markedly decreased the arrhythmia scores of AMI rats, indicating that SSYX suppresses ischemic arrhythmias to a certain extent. In addition, SSYX decreased the myocardial infarction area, which contributes towards protection of the myocardium and, ultimately, the reduction in the onset and severity of arrhythmias. The effect of SSYX on ameliorating myocardial ischemia damage could partially reverse left ventricular remodeling, reduce ischemic injury and prevent myocardial apoptosis.

The heartbeat is automatically initiated by electrical activity of cardiomyocytes at regular intervals. This process includes the formation of an action potential in a single cardiomyocyte and the spread of the action potential through the myocardium. An action potential reflects the processes of cardiac depolariza- 
tion and repolarization; it is based on the collaborative function of inward and outward currents through the ion channels of the cell membrane (29). Action potentials shorten rapidly during the first 2 min of AMI on account of the combined effects of components of the ischemic environment, including elevated extracellular $\mathrm{K}^{+}$, hypoxia, a low $\mathrm{pH}$, high partial pressure of carbon dioxide $\left(\mathrm{pCO}_{2}\right)$ and the accumulation of substances, such as catecholamines, resulting from the deprivation of blood flow. Each may exert an influence on membrane conductance $(30,31)$. Despite the electrophysiological mechanisms, cardiac re-entry has been regarded as an important mechanism causing malignant arrhythmias. In the present study, SSYX was shown to significantly prolong action potentials and slow cardiac repolarization. An appropriate extension of the action potential is conducive to increasing the refractory period and reducing myocardial excitability and re-entry (32). Therefore, the results of the present study suggested that SSYX altered the myocardial refractoriness and conductivity, and exerted a therapeutic effect on ischemic arrhythmias.

A moderate decrease in the resting potential from its normal value of -90 to $-80 \mathrm{mV}$ increases conduction velocity during AMI. A predominant cause of the decreased resting potential of ischemic cells is the elevated extracellular $\mathrm{K}^{+}$ and intracellular $\mathrm{Ca}^{2+}$, which result from a lack of blood flow. Ischemia and hypoxia interfere with ion channels, and predispose individuals to a disturbed cardiac rhythm (33). Cardiac $\mathrm{K}^{+}$channels determine the morphology of myocardial action potentials and the resting potential. $\mathrm{I}_{\mathrm{K} 1}$ is a strong inward rectification current, which stabilizes the membrane potential and is involved in the third period of action potential repolarization. $\mathrm{I}_{\text {to }}$ is the primary outward current of the cardiac rapid repolarization. Following depolarization, the inward sodium current $\left(\mathrm{I}_{\mathrm{Na}}\right)$ becomes inactivated, and $\mathrm{I}_{\mathrm{to}}$ initiates the rapid repolarization of the action potentials (34-36). Therefore, $I_{K}$ and $I_{t o}$ are both involved in cardiac repolarization, and are associated with cardiac excitability and arrhythmogenesis. In the present study, SSYX significantly decreased the densities of $\mathrm{I}_{\mathrm{to}}$, suppressed the first period of action potential repolarization and contributed to a prolongation of the myocardial action potential. Furthermore, inhibition of $\mathrm{I}_{\mathrm{to}}$ reduced the high irregularity of ischemic myocardial repolarization and the formation of re-entrant excitability, which helped to suppress re-entry and avoid the induction of Torsades de Pointes. Similarly, SSYX inhibited the inward current of $I_{K 1}$ significantly without affecting its reversal potential and rectification properties. This inhibitory effect was conductive to automatically eliminating the fourth period of action potential depolarization and inhibiting delayed afterdepolarization (DAD).

$\mathrm{Ca}^{2+}$ is involved in excitation-concentration coupling and $\mathrm{Ca}^{2+-}$ dependent signaling pathways. $\mathrm{Ca}^{2+}$ overload in ischemic myocytes results in cardiomyocyte death, myocardial ischemia injury and arrhythmias. $\mathrm{Ca}^{2+}$ overload is commonly associated with disorders of sarcoplasmic reticulum calcium stores and the L-type voltage-gated calcium channel, which consequently lead to DADs and EADs. Therefore, reducing $\mathrm{Ca}^{2+}$ overload would be beneficial for the treatment of myocardial ischemia and ischemic arrhythmias. However, prolongation of the action potentials may increase $\mathrm{Ca}^{2+}$ entry via the L-type calcium current $\left[\mathrm{I}_{\mathrm{Ca}(\mathrm{L})}\right]$ during the long plateau phase, which would cause an accumulation of $\mathrm{Ca}^{2+}$ in the sarcoplasmic reticulum, and spontaneous $\mathrm{Ca}^{2+}$ release from the sarcoplasmic reticulum. In the present study, SSYX significantly prolonged action potentials by inhibiting $\mathrm{I}_{\text {to }}$ and $\mathrm{I}_{\mathrm{K} 1}$ without affecting the intracellular $\mathrm{Ca}^{2+}$ concentration. In addition, SSYX alleviated the $\mathrm{Ca}^{2+}$ overload induced by $\mathrm{KCl}$ in cardiomyocytes, which helped to ameliorate the intracellular $\mathrm{Ca}^{2+}$ dysregulation of ischemic myocytes and improve cardiac function during ischemic arrhythmias. However, further studies are required in order to elucidate the precise mechanism by which SSYX regulates intracellular $\mathrm{Ca}^{2+}$.

Traditional pharmacology for TCM involves directly exposing cells, tissues or organs to the crude drug. However, due to the complexities of drug absorption and metabolism associated with TCM, the efficacy of the drug in its crude form may not be as good as the active ingredients after in vivo metabolism of the drug. Therefore, serum pharmacology for TCM was adopted in the present study. Serum pharmacology facilitates the direct application of active ingredients after the in vivo metabolism of the TCM, and utilizes cytological and molecular biological methods, thereby providing a conjunction of modern scientific technology and TCM research. In addition, observing and analyzing the processes of absorption and metabolism of drugs is beneficial in terms of eliminating the influence of various interference factors that are associated with in vitro experiments. Incubating myocytes with serum containing SSYX also corroborated previous findings on the efficacy of SSYX identified by means of in vivo and in vitro experiments. Serum pharmacology thus provides a novel approach for developing antiarrhythmic TCMs.

In conclusion, SSYX effectively prevents ischemic arrhythmias. In addition, SSYX is potent in extending action potentials and in inhibiting $\mathrm{I}_{\mathrm{to}}$ and $\mathrm{I}_{\mathrm{K} 1}$, thereby decreasing myocardial autorhythmicity and re-entrant excitability. Furthermore, SSYX alleviated myocardial $\mathrm{Ca}^{2+}$ overload, which contributes towards an elimination of DADs and a reduction in ultrastructural injuries of the myocardium. Therefore, SSYX may be used as an effective drug to treat ischemic arrhythmias.

\section{Acknowledgements}

This study was funded by the National Key Basic Research and Development Program (973 Program; grant no. 2012CB518606) and National Natural Science Foundation of China (grant no. 81100072).

\section{References}

1. Driessen HE, Bourgonje VJ, Van veen TA and Vos MA: New antiarrhythmic targets to control intracellular calcium handling. Neth Heart J 22: 198-213, 2014.

2. Gao FF, Hao SY, Huang ZQ, Zhang YM, Zhou YQ, Chen YC, Liu XP and Shi GG: Cardiac electrophysiological and antiarrhythmic effects of N-n-butyl haloperidol iodide. Cell Physiol Biochem 25: 433-442, 2010.

3. Davies MJ: Pathological view of sudden cardiac death. $\mathrm{Br}$ Heart J 45: 88-96, 1981.

4. Marbán E: Cardiac channelopathies. Nature 415: 213-218, 2002.

5. James RG, Arnold JM, Allen JD, Pantridge JF and Shanks RG: The effects of heart rate, myocardial ischemia and vagal stimulation on the threshold for ventricular fibrillation. Circulation 55: 311-317, 1977.

6. Fallavollita JA, Jacob S, Yong RF and Canty JM Jr: Regional alterations in SR $\mathrm{Ca}^{2+}$-ATPase, phospholamban and HSP-70 expression in chronic hibernating myocardium. Am J Physiol 277: H1418-H1428, 1999. 
7. Prunier F, Kawase Y, Gianni D, Scapin C, Danik SB, Ellinor PT, Hajjar RJ and Del Monte F: Prevention of ventricular arrhythmias with sarcoplasmic reticulum $\mathrm{Ca}^{2+}$ ATPase pump overexpression in a porcine model of ischemia reperfusion. Circulation 118: 614-624, 2008

8. Jiang XG, Jia JM and Li YS: Simultaneous determination of eight bioactive constituents in shensong yangxin capsule by UPLC. Chinese Herbal Medicines 5: 212-216, 2013.

9. Liu M, Li S, Zhao S, Wang H and Tu P: Studies on excretion kinetics of ten constituents in rat urine after oral administration of Shensong Yangxin Capsule by UPLC-MS/MS. Biomed Chromatogr 28: 525-533, 2014.

10. Jin ZY, Gong Q and Pu JL: The effect of Shensong Yangxin capsule on the porcine cardiac electrophysiology. Chinese Patent Medicine 31: 471-473, 2009.

11. Li N, Ma KJ, Wu XF, Sun Q, Zhang YH and Pu JL: Effects of Chinese herbs on multiple ion channels in isolated ventricular myocytes. Chin Med J (Engl) 120: 1068-1074, 2007.

12. Sun LP, Li N, Wu YL and Pu JL: Effects of Shensong Yangxin capsule on pacemaker channels encoded by human HCN4 gene. Chin Med J (Engl) 123: 3148-3150, 2010.

13. Vora A and Kulkami S: Pharmacotherapy to reduce arrhythmic mortality. Indian Heart J 66 (Suppl 1): S113-S119, 2014.

14. Yong SL and Wang QK: Animal models for cardiac arrhythmias. Methods Mol Med 129: 127-148, 2006.

15. Di Diego JM and Antzelevitch C: Ischemic ventricular arrhythmias: Experimental models and their clinical relevance. Heart Rhythm 8: 1963-1968, 2011.

16. Huggins CE, Bell JR, Pepe S and Delbridge LM: Benchmarking ventricular arrhythmias in the mouse-revisiting the 'Lambeth Conventions' 20 years on. Heart Lung Circ 17: 445-450, 2008.

17. Wu H, Tang Q, Yang J, Ye M and Dong W: Atorvastatin ameliorates myocardial ischemia/reperfusion injury through attenuation of endoplasmic reticulum stress-induced apoptosis. Int J Clin Exp Med 7: 4915-4923, 2014

18. Bochu W, Liancai Z and Qi C: Primary study on the application of serum pharmacology in Chinese traditional medicine. Colloid Surface B Biointerfaces 43: 194-197, 2005.

19. Graham EL, Balla C, Franchino H, Melman Y, del Monte F and Das S: Isolation, culture, and functional characterization of adult mouse cardiomyoctyes. J Vis Exp 24: e50289, 2013.

20. Gong DM, Shan HL, Zhou YH, Dong DL and Yang BF: The ion targets of arrhythmias induced by ouabain and aconitine in guinea pig and rat ventricular myocytes. Yao Xue Xue Bao 39: 328-332, 2004 (In Chinese).

21. Liang B, Nissen JD, Laursen M, Wang X, Skibsbye L, Hearing MC, Andersen MN, Rasmussen HB, Wickman K, Grunnet M, et al: G-protein-coupled inward rectifier potassium current contributes to ventricular repolarization. Cardiovasc Res 101: 175-184, 2014
22. Fang Z, Ren YP, Lu CY, Li Y, Xu Q, Peng L and Fan YY: Effects of sleep deprivation on action potential and transient outward potassium current in ventricular myocytes in rats. Med Sci Monit 21: 542-549, 2015.

23. Sun L, Ai J, Wang N, Zhang R, Li J, Zhang T, Wu W, Hang P, Lu Y and Yang B: Cerebral ischemia elicits aberration in myocardium contractile function and intracellular calcium handling. Cell Physiol Biochem 26: 421-530, 2010.

24. Billman GE: Novel Therapeutic targets for antiarrhythmic drugs. Wiley: Hoboken, New Jersey, 2010.

25. Sun J, Tan BK, Huang SH, Whiteman M and Zhu YZ: Effects of natural products on ischemic heart diseases and cardiovascular system. Acta Pharmacol Sin 23: 1142-1151, 2002.

26. Wang J, Li J and Feng B: Shen Song Yang Xin capsule combined with antiarrhythmic drugs, a new integrative medicine therapy, for the treatment of frequent premature ventricular contractions (FPVC): A-meta analysis of randomized controlled trials. Evid Based Complement Alternat Med 2014: 976713, 2014

27. Chai SB, Wang SR, Yao LF and Wu A: A study of the effect of Shensong Yangxin Capsule on ventricular remodeling after myocardial infarction and isolated heart action potential in rats. Beijing J Tradit Chin Med 12: 967-971, 2009 (In Chinese).

28. Wang AH, Pu JL, Qi XY, Miao WL, Hou ZS, Cong HL, Zhou JZ, Liu XF, Li SM, Han QH, et al: Evaluation of shensongyangxin capsule in the treatment of paroxysmal atrial fibrillation: A randomized, double-blind and controlled multicenter trial. Zhonghua Yi Xue Za Zhi 91: 1677-1681, 2011 (In Chinese).

29. Roden DM, Balser JR, George AL Jr and Anderson ME: Cardiac ion channels. Annu Rev Physiol 64: 431-475, 2002.

30. Isenberg G, Vereecke J, van der Heyden G and Carmeliet E: The shortening of the action potential by DNP in guinea-pig ventricular myocytes is mediated by an increase of a time-independent K conductance. Pflugers Arch 397: 251-259, 1983.

31. Vleugels A, Vereecke $\mathrm{J}$ and Carmeliet E: Ionic currents during hypoxia in voltage-clamped cat ventricular muscle. Circ Res 47: 501-508, 1980.

32. Wit AL and Coromilas J: Role of alterations in refractoriness and conduction in the genesis of reentrant arrhythmias. Implications for antiarrhythmic effects of class III drugs. Am J Cardiol 72: 3F-12F, 1993

33. Fox JJ, McHarg JL and Gilmour RF Jr: Ionic mechanism of electrical alternans. Am J Physiol Heart Circ Physiol 282: H516-H530, 2002.

34. Dhamoon AS and Jalife J: The inward rectifier current (IKl) controls cardiac excitability and is involved in arrhythmogenesis. Heart Rhythm 2: 316-324, 2005.

35. Anumonwo JM and Lopatin AN: Cardiac strong inward rectifier potassium channels. J Mol Cell Cardiol 48: 45-54, 2010.

36. Li GR and Dong MQ: Pharmacology of cardiac potassium channels. Adv Pharmacol 59: 93-134, 2010. 